\title{
Leg strength as a function of exposure to visual stimuli of different hues
}

\author{
ROBERT J. PELLEGRINI, ALEXANDER G. SCHAUSS, and THOMAS J. BIRK \\ San Jose State University, San Jose, California 95192
}

\begin{abstract}
Although the magnitude of effect was small, quadriceps femoris extension strength was found to be significantly $(p<.001)$ greater for 60 male subjects when they stared at a blue card than when they stared at a pink card. Hypothesized kinesiological effects of color are thus further indicated.
\end{abstract}

Recent developments in photobiology indicate that visual processing of light may, through biochemical mediating mechanisms, affect neurological and endocrinological functions (e.g., Hague, 1964; Kerenyi, 1977; Wurtman, 1975). Among the more provocative hypotheses generated by such findings is Ott's (1979) suggestion that physical strength is influenced by the electromagnetic properties of visual stimuli. With regard to particular colors, Ott argues that "pink or orange cause greater loss of strength and blue the least" (Ott, 1979 , p. 9). Considerable attention has been focused lately on applied implications of this hypothesis, especially in regard to possible tranquilizing effects of color in detention situations, in which a reduced incidence of aggression has been reported for inmates in correctional facility holding cells painted pink (Schauss, 1979).

Thus far, the available evidence for Ott's (1979) hypothesis concerning differential effects of color on muscle strength has been derived almost completely from highly subjective procedures such as the so-called "kinesoid" test. In this test, a subject holds his arm straight out in front of him and tries to resist as vigorously as possible a demonstrator's efforts to push the arm down. Following establishment of a "baseline," loss of strength is typically shown when the test is repeated immediately while a pink card is held about 15 in. in front of the subject's eyes; a recovery in resistance is observed when a blue card is then similarly placed (Schauss, 1979). As noted elsewhere (Pellegrini \& Schauss, 1980), conclusions drawn from such demonstrations are tentative at best, largely because the strength measure is based entirely upon impressions of the subject's resistance. Vulnerability to confounding by judgmental subjectivity is increased by the demonstrator's considerable leverage advantage over even the strongest subject.

In the only rigorous experimental test yet conducted of the color-strength hypothesis, significantly greater hand-dynamometer squeeze scores were found for

Requests for reprints should be sent to Robert J. Pellegrini, Department of Psychology, San Jose State University, San Jose California 95192. Alexander Schauss is now the director of the Institute for Biosocial Research, Tacoma, Washington 98466. subjects as they stared at a blue, compared with a pink, cardboard plate (Pellegrini \& Schauss, 1980). The magnitude of effect, however, was so limited as to leave the issue very much in doubt. The present study was thus designed to extend experimental inquiry along these lines to measurement of strength in a large skeletal muscle group.

\section{METHOD}

\section{Subjects}

The subjects were 60 male students in a general psychology course who participated as part of a course research requirement.

\section{Materials}

Equipment used was as follows: a padded wooden table with cable and hook attachments for kinesiological assessment purposes, a double-armed goniometer, one pink and one blue $18 \times 24$ in. cardboard plate, a specially constructed tubular viewframe apparatus designed to hold the cardboard plates in front of a subject's eyes, a cable tensiometer, and a stopwatch.

\section{Procedure}

Upon arriving at the exercise physiology laboratory, the subject was told the experiment involved tests of strength under different visual conditions. He was seated with the back of his knees against the front end of the table, and his right ankle was placed into a 2 -in.-wide strap connected to a cable attached to a hook in the center floorpiece of the table. The strap was placed over the top of the subject's ankle joint at the point at which the upper part of the foot and the lower leg join at an approximately 90-deg angle, and the cable was hooked so that this placement of the strap was maintained when the subject's upper and lower leg formed a 90-deg angle at the knee joint. The latter angle was measured with the goniometer. This arrangement allowed for assessment of strength due primarily to the quadriceps femoris group in the upper leg (Wells \& Luttgens, 1976). The subject's hands were placed about 6 in. from the end of the table, and the subject was told to hold onto the table in just that position throughout the experiment.

The viewframe was then set in place over the subject's head; it rested on the subject's shoulders and chest. Next, one of the cardboard plates was inserted into the viewframe, 12-15 in. in front of the subject's eyes, and the subject was instructed to stare at the middle of the card until told to stop. After exactly $60 \mathrm{sec}$, the subject was told to continue staring at the card and to extend his right leg (the one in the strap) as hard as he possibly could. The experimenter measured this maximumextension response by affixing the tensiometer to the ankle strap cable. The first card was then removed from the viewframe, 
and the subject was told to rest for $1 \mathrm{~min}$. Following a $60-\mathrm{sec}$ rest period, this procedure was repeated for the other color plate. Until presented in the viewframe, both plates were concealed from the subject. Order effects were controlled by randomly assigning 30 subjects to a pink-blue presentation order and 30 subjects to a blue-pink presentation order.

\section{RESULTS AND DISCUSSION}

In line with Ott's (1979) hypothesis, a two-tailed $t$ test for matched samples indicated significantly higher $[\mathrm{t}(59)=3.88, \mathrm{p}<.001]$ maximum quadriceps femoris extension scores during exposure of the blue (mean $=$ $105.95 \mathrm{lb}, \mathrm{SD}=26.34$ ) than during exposure of the pink $($ mean $=102.23 \mathrm{lb}, \mathrm{SD}=28.10)$ stimulus card. In view of the power of the statistical test used and the rather small mean difference between plate color conditions, a magnitude-of-effect estimate (Hays, 1963) was also calculated. This procedure yielded an estimated $\omega^{2}=.11$, indicating that the plate color variable accounted for about $11 \%$ of the variance on the dependent measure of extension strength.

As in the first experiment (Pellegrini \& Schauss, 1980), subjects showed a significantly greater strength response while visually focused on a blue stimulus than while focused on a pink stimulus. Again, magnitude of effect was found to be quite small. But effect size here was more than twice as large as the $5 \%$ estimate obtained in the previous experiment, which tested grip streingth in the preferred hand; this constitutes a $120 \%$ increase in the amount of variance accounted for by the color manipulation. The more robust color effect obtained here may thus reflect a greater susceptibility to visualhue-difference manipulation of strength in large muscle groups such as those in the leg than in smaller groups such as those in the arm and hand. This puzzling matter remains to be explored further.

It is tempting to speculate as to psychological (e.g., infant gender identity and sex-role stereotypical associations to pink and blue) and/or physiological (e.g., neuroendocrine mechanisms linked to the visual system) processes underlying the observed effects. But such conjecture seems premature until reliability of the presumed effects and the conditions under which they may be shown most powerfully have been more unambiguously established. Even from a most conservative perspective on inference, however, the results of the present study must be acknowledged as additional supporting evidence for the kinesiological effects of color.

\section{REFERENCES}

Hague, E. G. (Ed.). Photo-neuro-endocrine effects in circadian systems, with particular reference to the eye. Annals of the New York Academy of Sciences, 1964, 117, 1-645.

HAYS, W. L. Statistics for psychologists. New York: Holt, 1963.

KerenYi, N. A. The pineal gland: What is its true importance? Modern Medicine, 1977, 45, 81-84.

OTт, J. N. The dual function of the eyes. Southern Journal of Optometry, 1979, 21, 8-13.

Pellegrini, R. J., \& Schauss, A. G. Muscle strength as a function of exposure to hue differences in visual stimuli: An experimental test of the kinesoid hypothesis. Journal of Orthomolecular Psychiatry, 1980, 9, 144-147.

Schauss, A. G. Tranquilizing effect of color reduces aggressive behavior and potential violence. Journal of Orthomolecular Psychiatry, 1979, 8, 218-220.

Wells, K. F., \& Luttgens, K. Kinesiology. Philadelphia: Saunders, 1976.

Wurtman, R. J. The effects of light on the human body. Scientific American, 1975, 233, 68-77.

(Received for publication June 30, 1980.) 